\title{
and Virtue Toward Excellence
}

\author{
Paul B. Baltes and Ursula M. Staudinger
}

Max Planck Institute for Human Development

The primary focus of this article is on the presentation of wisdom research conducted under the heading of the Berlin wisdom paradigm. Informed by a cultural-historical analysis, wisdom in this paradigm is defined as an expert knowledge system concerning the fundamental pragmatics of life. These include knowledge and judgment about the meaning and conduct of life and the orchestration of human development toward excellence while attending conjointly to personal and collective well-being. Measurement includes think-aloud protocols concerning various problems of life associated with life planning, life management, and life review. Responses are evaluated with reference to a family of 5 criteria: rich factual and procedural knowledge, lifespan contextualism, relativism of values and life priorities, and recognition and management of uncertainty. A series of studies is reported that aim to describe, explain, and optimize wisdom. The authors conclude with a new theoretical perspective that characterizes wisdom as a cognitive and motivational metaheuristic (pragmatic) that organizes and orchestrates knowledge toward human excellence in mind and virtue, both individually and collectively.

W isdom is generally considered the pinnacle of insight into the human condition and about the means and ends of a good life (P. Baltes, Smith, \& Staudinger, 1992; Kekes, 1995; Staudinger \& Baltes, 1996b). In the positive-psychology spirit of this special issue of the American Psychologist, our interest in wisdom has been spurred by a motivation to identify and highlight the best of what society and humans can accomplish concerning their own development and that of others. As has been true several times throughout the millennia ( $\mathrm{P}$. Baltes, 1999; Kekes, 1995; Rice, 1958), the current scholarly discourse about the structure and function of wisdom evinces another period of rejuvenation. Occasionally, researchers argue that such historical periods of rejuvenation follow the principle of societal need for reflection about its own attainments, status, and future direction.

The purpose of this article is twofold. First and most important, we present an overview of our work on the psychology of wisdom. Proceeding from a general theoretical framework, we have developed an empirical research paradigm to study wisdom (P. Baltes, Dittmann-Kohli, \&
Dixon, 1984; P. Baltes \& Smith, 1990; P. Baltes et al., 1992; P. Baltes \& Staudinger, 1993; Dittmann-Kohli \& Baltes, 1990; Dixon \& Baltes, 1986; Smith \& Baltes, 1990; Staudinger \& Baltes, 1996b). Second, to embed our work in a larger context, we begin by summarizing briefly the work of other psychologists interested in the topic of wisdom (see also, Clayton \& Birren, 1980; Holliday \& Chandler, 1986; Orwoll \& Perlmutter, 1990; Pasupathi \& Baltes, in press; Staudinger \& Baltes, 1994; Sternberg, 1990, 1998; Taranto, 1989).

Historically, it has been mainly the fields of philosophy and religious studies that have served as the central forum for discourse about the concept of wisdom (Assmann, 1994; P. Baltes, 1993, 1999; Kekes, 1995; Oelmüller, 1989; Robinson, 1990; Rudolph, 1987; Welsch, 1995). For the current historical moment, however, renewed interest in the topic of wisdom is evident in a wide spectrum of disciplines, ranging from the traditional mentors of wisdom, philosophy, and religious studies, to cultural anthropology, political science, education, and psychology (Agazzi, 1991; Arlin, 1993; Assmann, 1994; P. Baltes, 1993, 1999; Cook, 1993; Lehrer, Lum, Slichta, \& Smith, 1996; Maxwell, 1984; Nichols, 1996; Nozick, 1993; Staudinger \& Baltes, 1996b; Sternberg, 1990; Welsch, 1995).

Because of the culturally rich meaning and heritage of wisdom, defining and operationalizing the concept of wisdom as a scientifically grounded psychological construct is

Paul B. Baltes and Ursula M. Staudinger, Center for Lifespan Psychology, Max Planck Institute for Human Development, Berlin, Germany.

Ursula M. Staudinger is now at the Department of Psychology, Dresden University of Technology, Dresden, Germany.

We would like to acknowledge the many contributions of earlier colleagues in the Wisdom Project at the Max Planck Institute for Human Development. Earlier collaborators included, foremost, Freya DittmannKohli, Roger Dixon, and Jacqui Smith. We also thank Monisha Pasupathi for her valuable comments.

We thank especially Irmgard Pahl, who, as editorial assistant, has participated with the utmost competence, care, and curiosity in examining cultural-historical and philosophical works regarding their treatment of wisdom.

Correspondence concerning this article should be addressed to Paul B. Baltes, Lifespan Psychology, Max Planck Institute for Human Development, Lentzeallee 94, 14195 Berlin, Germany. Electronic mail may be sent to sekbaltes@mpib-berlin.mpg.de or to staudinger@psyl.psych.tudresden.de. 


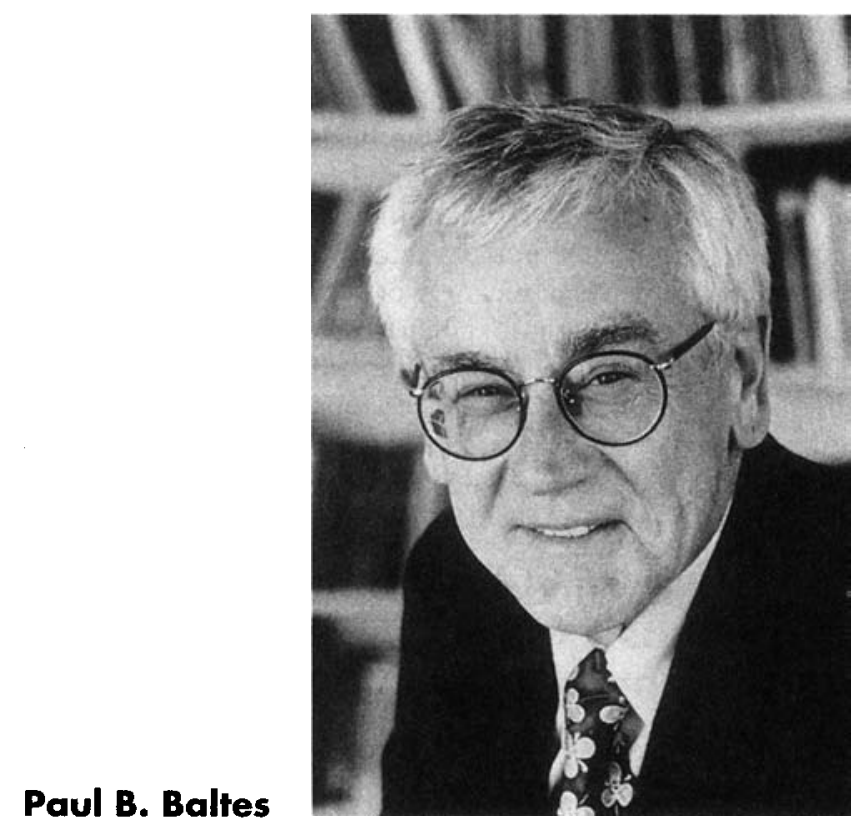

not easy. Wisdom may be beyond what psychological methods and concepts can achieve. The first president of the American Psychological Association, G. Stanley Hall (1922), was one of the first psychologists to tackle this task, originally in an anonymous article published in 1921 in the Atlantic Monthly. Subsequently, it was primarily the lifespan model of Erikson (1959; Clayton \& Birren, 1980; McAdams \& de St. Aubin, 1998) and the emergence of lifespan psychology (P. Baltes, Staudinger, \& Lindenberger, 1999) that kept wisdom in the domain of psychological analysis.

It was not until the 1980s that a more diverse group of psychological scholars and researchers began to engage themselves with the topic of wisdom, although most work was theoretical rather than empirical. A book edited by Sternberg (1990) was a signal of this growing interest in wisdom, as are entries on wisdom in a variety of behavioral-science-oriented encyclopedias (e.g., P. Baltes \& Staudinger, 1998; Pasupathi \& Baltes, in press; Staudinger \& Baltes, 1994).

\section{Implicit and Explicit Psychological Theories of Wisdom}

Not surprisingly, because of the multidisciplinary nature of the wisdom concept, including its integrative feature of linking mind to virtue, psychological research on wisdom is multifaceted. Aside from issues such as the nature of methodology applied and the content range that is assigned to the psychological sphere of wisdom (Birren \& Fisher, 1990), two major branches can be distinguished: implicit theories and explicit theories of wisdom (Sternberg, 1990).

\section{Implicit Theories}

The first branch of this sphere, implicit theories of wisdom, consists of psychological research that is associated with folk-psychological or common-sense approaches, a line of work initiated by Clayton (Clayton \& Birren, 1980), Holliday and Chandler (1986), Sternberg (1985, 1986), Orwoll and Perlmutter (1990), and Sowarka (1989). At stake here is the question of how the term wisdom is used in everyday language and how wise persons are characterized.

In our assessment, results on implicit conceptions of wisdom and wise persons permit five conclusions about the concept of wisdom: (a) Wisdom is a concept that carries specific meaning that is widely shared and understood in its language-based representation. For example, wisdom is clearly distinct from other wisdom-related psychological concepts such as social intelligence, maturity, or creativity. (b) Wisdom is judged to be an exceptional level of human functioning. It is related to excellence and ideals of human development. (c) Wisdom identifies a state of mind and behavior that includes the coordinated and balanced interplay of intellectual, affective, and motivational aspects of human functioning. (d) Wisdom is viewed as associated with a high degree of personal and interpersonal competence, including the ability to listen, evaluate, and to give advice. (e) Wisdom involves good intentions. It is used for the well-being of oneself and others.

In many ways, as is true for many achievements of human development (Cole, 1996; Shweder, 1991), such implicit and folk-psychological characterizations of wisdom are mainly the product of cultural history and its impact on current society (see also, Csikszentmihalyi \& Rathunde, 1990). There is a saying that states, "Cultural memory is the mother of wisdom." Individuals partake in this culture-produced concept of wisdom.

Consistent with this view, a more comprehensive characterization of wisdom can be deduced from culturalhistorical and philosophical analyses of the wisdom concept (Assmann, 1994; P. Baltes, 1993, 1999; Kekes, 1995; Lehrer et al., 1996). To illustrate, P. Baltes (1993, 1999, see Appendix A) identified seven properties of wisdom that emerge when analyzing and synthesizing cultural-historical and philosophical work: (a) Wisdom represents a truly superior level of knowledge, judgment, and advice; (b) wisdorn addresses important and difficult questions and strategies about the conduct and meaning of life; (c) wisdom includes knowledge about the limits of knowledge and the uncertainties of the world; (d) wisdom constitutes knowledge with extraordinary scope, depth, measure, and balance; (e) wisdom involves a perfect synergy of mind and character; that is, an orchestration of knowledge and virtues; (f) wisdom represents knowledge used for the good or well-being of oneself and that of others; and (g) wisdom, although difficult to achieve and to specify, is easily recognized when manifested.

When comparing the results of modern-day research on subjective beliefs of wisdom (see above) with these more general perspectives on wisdom that can be deduced from philosophical and cultural-historical analyses, there is much overlap (P. Baltes, 1999). If anything, however, the philosophical-historical analysis of wisdom is more general, as it integrates the beliefs held by many individuals into a common set of properties about which there is much 


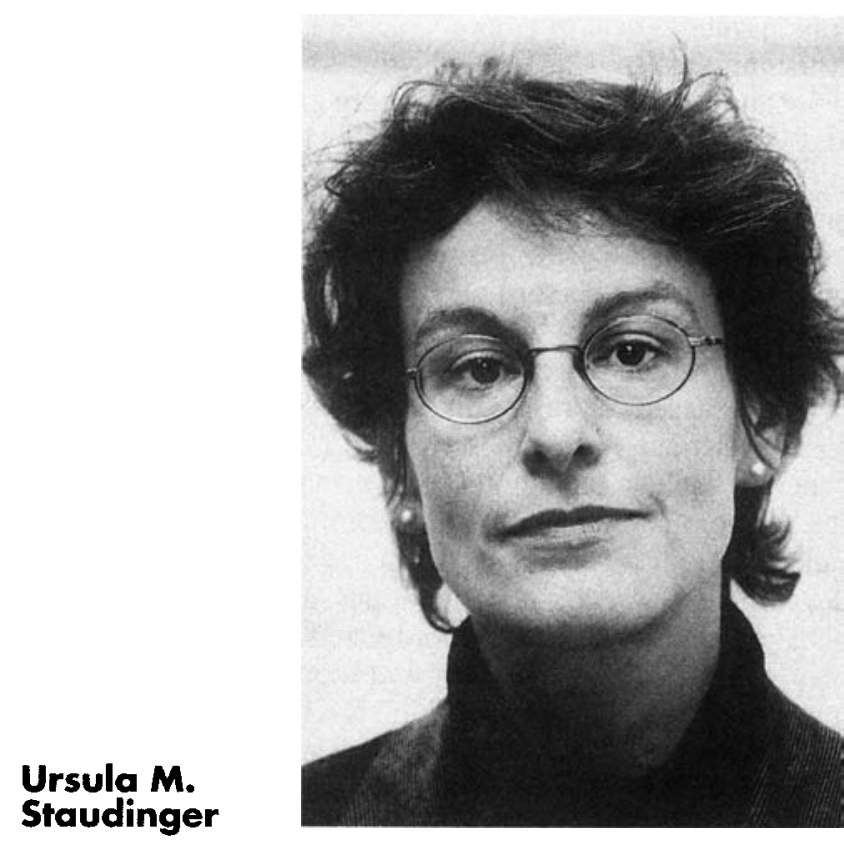

collective and scholarship-based intersubjectivity. The beliefs of single individuals, in other words, are usually less developed (comprehensive and organized) than those offered by philosophical and cultural-historical analyses.

\section{Explicit Theories}

Explicit psychological theories of wisdom go beyond the characterization of wisdom and a wise person in terms of language-based descriptions. They focus on behavioral manifestations or expressions of wisdom. In psychology, such explicit theories of wisdom refer to theoretical constructions of wisdom that lend themselves to empirical inquiry in terms of quantifiable operationalization as well as the identification of relevant antecedents, correlates, and consequences of wisdom and wisdom-related concepts.

Implicit and explicit psychological theories of wisdom are intertwined of course. For example, the information provided by implicit theories of wisdom and the culturalhistorical work on wisdom offers a frame within which explicit psychological work can be evaluated. Specifically, one can ask whether explicit and behavior-oriented work on the psychology of wisdom sufficiently agrees with the language-based construction of wisdom as reflected in cultural history, philosophy, and folk psychology.

Theoretical and empirical work on explicit psychological theories of wisdom can be roughly divided into three groups: (a) the conceptualization of wisdom as a personal characteristic or constellation of personality dispositions (e.g., Erikson, 1959; McAdams \& de St. Aubin, 1998), (b) the conceptualization of wisdom in the neo-Piagetian tradition of postformal and dialectical thought (e.g., Alexander \& Langer, 1990; Labouvie-Vief, 1990), and (c) the conceptualization of wisdom as an expert system dealing with the meaning and conduct of life (P. Baltes \& Smith,
1990; Dittmann-Kohli \& Baltes, 1990; Staudinger \& Baltes, 1994).

This third category of explicit theories guides our own empirical work and serves as the basis for the psychological paradigm of wisdom presented in the following. For another well-elaborated psychological theory of wisdom (although largely theoretical rather than empirical), the reader can refer to a recent work by Sternberg (1998). Specifically, Sternberg conceptualized wisdom as the application of tacit knowledge toward the achievement of a common good through a balance among multiple personal (intra-, inter-, and extrapersonal) interests and environmental conditions. There is much similarity between our respective theories.

\section{The Berlin Wisdom Paradigm: Wisdom as Expertise in the Fundamental Pragmatics of Life}

To prevent a possible misunderstanding, we begin by making explicit that our own empirical approach is only one way to operationalize our general perspectives on wisdom (see Appendix A and Figure 1). We do not argue that this line of empirical operationalization covers the entire meaning space of wisdom. Wisdom as a theoretical and cultural construct is more than any given empirical method can achieve.

Because of the emphasis of wisdom on excellence, we define wisdom as an expertise in the conduct and meaning of life. In this vein, wisdom is a key factor in the construction of a "good life" (M. Baltes \& Carstensen, 1996; P. Baltes et al., 1992; Staudinger, 1999b). An important step toward the further explication of this definition of wisdom as expertise was a specification of the content that can properly be said to fall within the category of wisdom (cf. P. Baltes \& Smith, 1990; Baltes \& Staudinger, 1993). To this end, we introduced the concept of the fundamental pragmatics of life. With fundamental pragmatics, we mean knowledge and judgment about the essence of the human condition and the ways and means of planning, managing, and understanding a good life.

Included in the fundamental pragmatics of life are, for example, knowledge about the conditions, variability, ontogenetic changes, and historicity of life development as well as knowledge of life's obligations and life goals; understanding of the socially and contextually intertwined nature of human life, including its finitude, cultural conditioning, and incompleteness; and knowledge about oneself and the limits of one's own knowledge and the translation of knowledge into overt behavior. Equally central to wisdom-related knowledge and judgment are the "spiritual" incomprehensibilities of life, such as the mind-body dynamics or the existence of a divine being.

These examples illustrate that the territory of inquiry that we label as the fundamental pragmatics of life is very different from other domains that have been identified in research on expertise. For the most part, past research on expertise has concentrated on well-defined systems of factual and procedural knowledge such as physics or chess. 
Figure 1

A Research Framework Describing Antecedent Factors and Mediating Processes for the Acquisition and Maintenance of Wisdom-Related Knowledge and Skills Across the Life Span

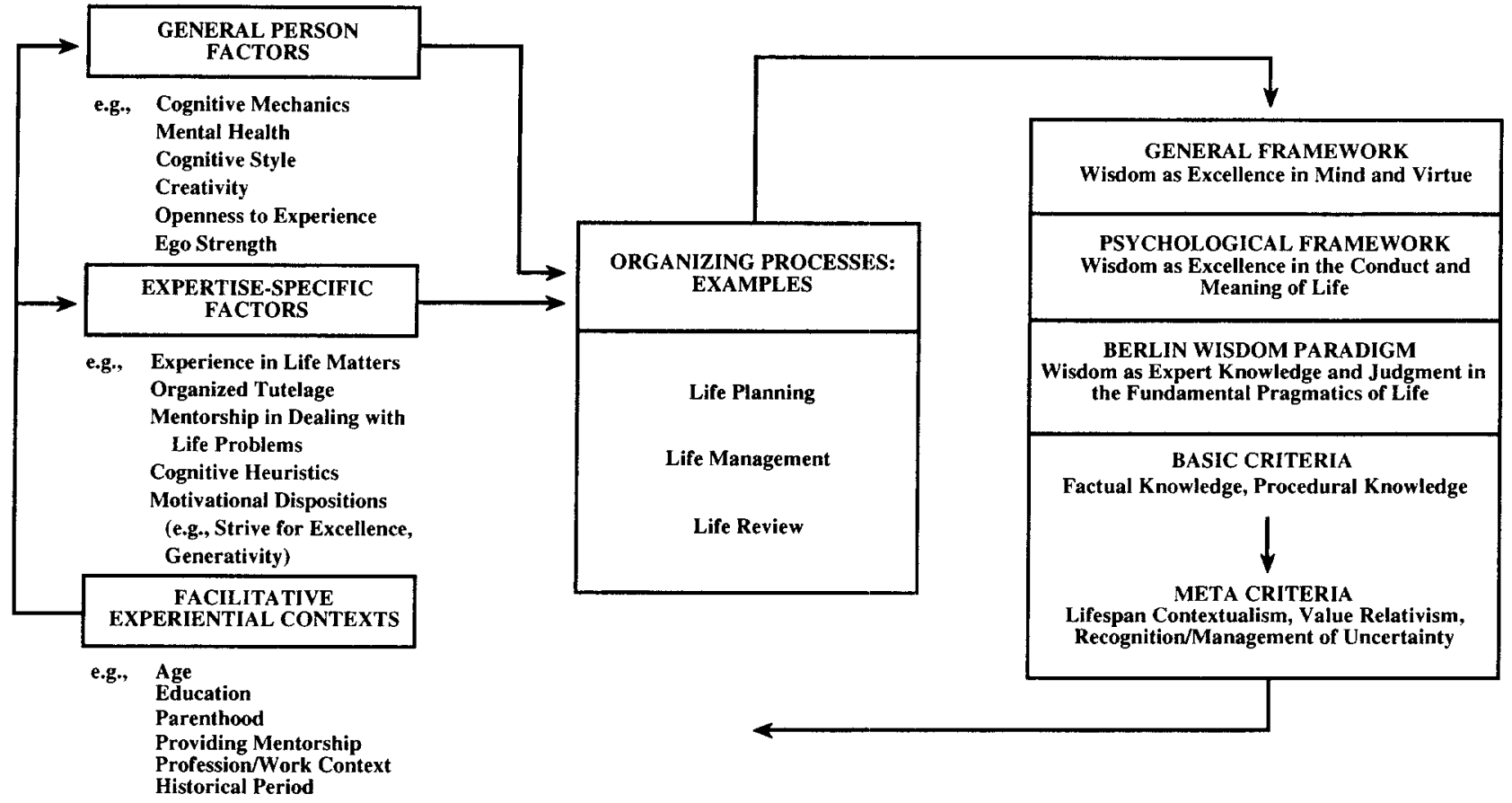

Note. The likelihood of attaining expert levels of performance in this prototypical domain of the cognitive pragmatics of the mind is assumed to depend on an effective coalition of life-context, expertise-specific, and general person-related factors.

Wisdom, contrariwise, is an area that in itself represents an open and ill-defined body of knowledge. Nonetheless, we assume that wisdom has a core and that wisdom-related manifestations, if and when they occur, can be evaluated in terms of indicators of quality and quantity. Our empirical research results support this assumption. Many people, after some training, are capable of reaching a high consensus in the evaluation of wisdom-related products of performances.

\section{A Family of Five Criteria for Assessing the Quality of Wisdom-Related Performance}

In our work, the quality of wisdom and the capacity for judgment in the fundamental pragmatics of life are defined through a set of five criteria listed in the bottom part of Appendix A. As described in more detail elsewhere (e.g., P. Baltes et al., 1984, 1992; Dittmann-Kohli \& Baltes, 1990; Smith \& Baltes, 1990; Staudinger \& Baltes, 1996a, 1996b), this set of criteria builds on the theoretical and empirical approaches mentioned above: that is, research on expertise, lifespan psychology of cognition and personality, the neo-Piagetian tradition of adult cognitive development, as well as cultural-historical analyses of wisdom.

The two general, basic wisdom criteria (factual and procedural knowledge) are characteristic of all types of expertise and stem from the tradition of research in exper- tise. Applied to the present subject area, these criteria are (a) rich factual (declarative) knowledge about the fundamental pragmatics of life and (b) rich procedural knowledge about the fundamental pragmatics of life. The factual knowledge part concerns knowledge about such topics as human nature, life-long development, variations in developmental processes and outcomes, interpersonal relations, social norms, critical events in life and their possible constellations, as well as knowledge about the coordination of the well-being of oneself and that of others. Procedural knowledge about the fundamental pragmatics of life involves strategies and heuristics for dealing with the meaning and conduct of life-for example, heuristics for giving advice and for the structuring and weighing of life goals, ways to handle life conflicts and life decisions, and knowledge about alternative back-up strategies if development were not to proceed as expected.

In addition to these two basic criteria, we have formulated three meta-criteria that in their separate and joint expression, we consider specific for wisdom. These criteria stem primarily (but not entirely) from the lifespan psychology of cognition and personality (e.g., Alexander \& Langer, 1990; P. Baltes, 1987, 1997; P. Baltes et al., 1999). The first metacriterion, lifespan contextualism, is meant to identify knowledge that considers the many themes and 
contexts of life (e.g., education, family, work, friends, leisure, the public good of society, etc.), their interrelations and cultural variations, and in addition, incorporates a lifetime temporal perspective (i.e., past, present, and future). Another feature of lifespan contextualism is the historical and social location of individual lifespan development as well as the idiographic or nonnormative events that operate in human development (Bandura, 1982).

The second wisdom-specific metacriterion, relativism of values and life priorities, deals with the acknowledgment of and tolerance for value differences and the relativity of the values held by individuals and society. Wisdom, of course, is not meant to imply full-blown relativity of values and value-related priorities. On the contrary, it includes an explicit concern with the topic of virtue and the common good. However, aside from the recognition of certain universal values (Kekes, 1995), value-relative knowledge, judgment, and advice are part of the essence of wisdom.

The third meta-criterion, the recognition of and management of uncertainty, is based on the ideas (e.g., Baron, 1988; Dawes, 1988; Gigerenzer, 1996; Nisbett \& Ross, 1980; Simon, 1983; Stich, 1990) that (a) the validity of human information processing itself is essentially limited (constrained), (b) individuals have access only to select parts of reality, and (c) the future cannot be fully known in advance. Wisdom-related knowledge and judgment are expected to offer ways and means to deal with such uncertainty about human insight and the conditions of the world, both individually and collectively.

For space limitations, we are not able to explicate how our family of wisdom criteria relates to work conducted by others who are also engaged in the study of wisdom or related topics. Aside from Sternberg's (1998) important recent effort mentioned earlier, with its focus on tacit knowledge dealing with a balanced integration of intra-, inter-, and extrapersonal interests, we note especially works by Arlin (1993) on the ability of mature thinkers to identify problems, by Dörner (1983) on complex problem solving, by Kitchener and Brenner (1990) on the concept of tolerance for ambiguity, by Eriksonian researchers on generativity and other gains of adulthood (McAdams \& de St. Aubin, 1998), by researchers interested in the self-based regulation of cognition and emotion during adulthood (Blanchard-Fields \& Hess, 1996; Carstensen, 1995; Labouvie-Vief, 1995), and conceptual and empirical work by Riegel (1973) and Basseches (1984) on dialectical thinking as a postformal mode of adult reasoning. Such lines of inquiry are very relevant, and we have benefited from their consideration. In the context of our own approach, however, perhaps with the exception of Sternberg (1998), these various approaches each represent only one important component or facet of the wisdom-related domain of expertise that we attempt to articulate and study.

\section{The Empirical Assessment of Wisdom-Related Performance}

The five qualitative criteria for the evaluation of wisdomrelated knowledge and judgment are suited for application to a wide array of person-specific as well as social manifestations of wisdom. This topical array ranges from state constitutions or works in the religious sphere on spirituality, to personal documents such as biographies and autobiographies, to the way people assess and respond to tasks of life planning, life management, and life review, whether it be their own or that of another. Wisdom is located in many sources and to achieve its highest level of manifestation, it is likely that these sources need to be interrelated and used as an ensemble.

In our work, we primarily have focused on, to date, searching for manifestations of wisdom in individual minds by asking people to respond to various problems of life (for a more detailed description, see, e.g., Staudinger \& Baltes, 1996a). Specifically, and as illustrated in Appendix B, study participants are confronted under standardized conditions with difficult life problems of fictitious people, such as the following example: "Someone receives a telephone call from a good friend who says that he or she cannot go on like this and has decided to commit suicide. What might one/the person take into consideration and do in such a situation?" Another example is as follows: "In reflecting over their lives, people sometimes realize that they have not achieved what they had once planned to achieve. What should they do and consider?" The participants are then asked to reflect out loud on the presented dilemma.

The responses are recorded on tape and transcribed. Before the tasks are administered, participants are given practice in thinking aloud (Ericsson \& Simon, 1984) and thinking about a hypothetical person. Appendix B contains an excerpt from a high- and low-rated response to the question of what to consider and do in the case of a 15-year-old girl who wants to get married right away.

For the purpose of obtaining quantified scores, a select panel of judges, who are extensively trained and calibrated in applying the criteria, evaluates the protocols of the respondents in light of the five wisdom-related criteria using a 7 -point scale. The training proceeds on the basis of a manual. The reliability of this rating method is very satisfactory. In the empirical research conducted so far, the intercorrelation between the five criteria has always been high, approaching values between .50 and .77 ; test-retest correlations over 12 months range in adults between 65 and .94; and the multidimensional measurement space that is based on multiple tasks of wisdom conforms to the five-criterion framework outlined (P. Baltes \& Staudinger, 1993: Staudinger, Raykov, Böhmig-Krumhaar, \& Baltes, 1999).

In general, we speak of a "wise" protocol only if it has received a high rating in all five areas (e.g., a rating greater than 5 for each criterion on the 7-point scale). As is the case in research on other expert systems, it is an open question to what degree the development of wisdom reflects the accumulation of quantity or also the acquisition of new qualities. Our general approach, which is consistent with many cultural-historical views of wisdom (P. Baltes, 1999), is to view wisdom as more or less (quantitative) a 
phenomenon without excluding the possibility that its final achievement is a qualitatively new step.

\section{Antecedents, Correlates, and Consequences of Wisdom}

Treating wisdom as an expert system associated with the fundamental pragmatics of life suggests a number of conditions under which wisdom is likely to develop (Bloom, 1985; Ericsson \& Lehmann, 1996). Foremost are four theoretical perspectives. First, like any expertise, the acquisition and refinement of wisdom involves an extended and intense process of learning, practice, as well as the motivation to strive toward excellence. Second, wisdom is a complex and multifaceted phenomenon. Therefore, a variety of micro- and macrofactors and processes need to collaborate to generate wisdom. Third, because of the integrative aspects of wisdom in linking knowledge with virtue, it is likely that the antecedents of wisdom are grounded in the orchestration of several characteristics: cognitive, motivational, social, interpersonal, and spiritual. Fourth, as with any other high-level expertise, guidance by mentors or other wisdom-enhancing "voices" of society as well as the experience and mastery of critical life experiences are likely necessary.

Figure 1 summarizes some of our analytic efforts at translating these general theoretical perspectives into a testable framework. The framework describes a series of ontogenetic conditions and processes that, as distant and proximal factors, need to work together "synergetically" so that something like wisdom can develop. Specifically, we distinguish three categories of conditions that are relevant to the development of wisdom: (a) general personal characteristics, (b) characteristics and experiential contexts that are specific to the acquisition of expertise in the area of the fundamental pragmatics of life, and (c) macrostructural contexts that are linked to certain constellations of wisdomrelated experience. Moving toward wisdom requires some orchestrated coalition of these factors. It is likely, however, that there is not a single pathway; rather, we proceed in our work with the idea of multiple but constrained pathways to wisdom.

Because of the visibility of Erikson's (1959) theory of wisdom (e.g., Clayton \& Birren, 1980; McAdams \& de St. Aubin, 1998), we add some observations to prevent possible misunderstandings and to differentiate our own conception from his. In our view, the Eriksonian approach to wisdom, with its lifespan-, self-, and motivation-based conception of wisdom, provides one important set of constellations that we consider critical for the understanding of wisdom. However, our conception of wisdom differs in significant ways. First, Erikson's theory does not explicate many of the other expertise-related factors and processes that, in addition to personality-related factors, are at the foundation of the acquisition and refinement of wisdom. Second, our conception of wisdom entails more than the mind and personality of individuals. In our conception, wisdom is fundamentally a cultural and collective product in which individuals participate. Individuals are only some of the carriers and outcomes of wisdom. Third, the sub- stantive content of Erikson's wisdom theory, with its primary emphasis on self-referenced integrity and generativity, represents but a subset of the territory that we propose to demarcate as wisdom. Other subsets involve, for instance, the heuristics of knowledge organization and decision making that are associated with wisdom-related behavior.

At the center of the ontogenetic schema (cf. Figure 1), we highlight some of the processes that we consider as the perpetual and organizing regulators of the development of wisdom. Finally, on the right-hand side of Figure 1, there is a schematic presentation of the inferential processes that we engaged in as we translated the general culturally and philosophically legitimated conception of wisdom into our specific psychological operationalization. In the lower part of the right column, assumptions about the sequential course of development of the five criteria for wisdom are suggested. In line with the model for the development of expertise suggested by Anderson (1987), for example, we propose that in the course of development of expertise, a shift of emphasis takes place from declarative (factual) to procedural knowledge. From this foundation, we expect the body of wisdom-constitutive knowledge to emerge that is captured with the three metacriteria: lifespan contextualism, relativism in values and life priorities, and recognition and management of uncertainty.

We have and are considering adding to this family of five criteria another feature of wisdom. We now think that it may be important to make more explicit the motivational-emotional orientation associated with the use of wisdom, that is, that wisdom is (a) intended for the wellbeing of oneself and others and (b) involves an effective coordination of mind and virtue. So far, we had included this motivational-emotional aspect of wisdom as a correlate of practically all criteria. Such an approach, however, may not be explicit enough and, therefore, could falsely generate the impression that our model does not consider motivational-emotional dimensions and the notion that wisdom deals with the personal and common good or well-being. Nothing could be further from our intentions.

\section{Empirical Findings: Berlin Wisdom Paradigm}

In the following, we summarize some of the main findings obtained when translating our paradigm into empirical research. Studies in which we confronted individuals with the kind of life problems described in Appendix B are most prominent. More recently, we have added to this approach an additional methodological and conceptual frame. In this new facet of research, we focused on a theory of successful lifespan development that defines the three processes of selection, optimization, and compensation as the key elements leading to developmental advances (M. Baltes \& Carstensen, 1996; P. Baltes, 1997; Freund \& Baltes, in press; Marsiske, Lang, Baltes, \& Baltes, 1995). We considered knowledge about these processes as part of the domain of wisdom (P. Baltes et al., 1992). In this work, we used proverbs to examine whether individuals had knowl- 
Figure 2

Adult Developmental Age Gradients for Prototypical Measures of the Cognitive Mechanics (Working Memory; Lindenberger \& Baltes, 1997) and the Cognitive Pragmatics (Wisdom; Staudinger \& Baltes, 19966)
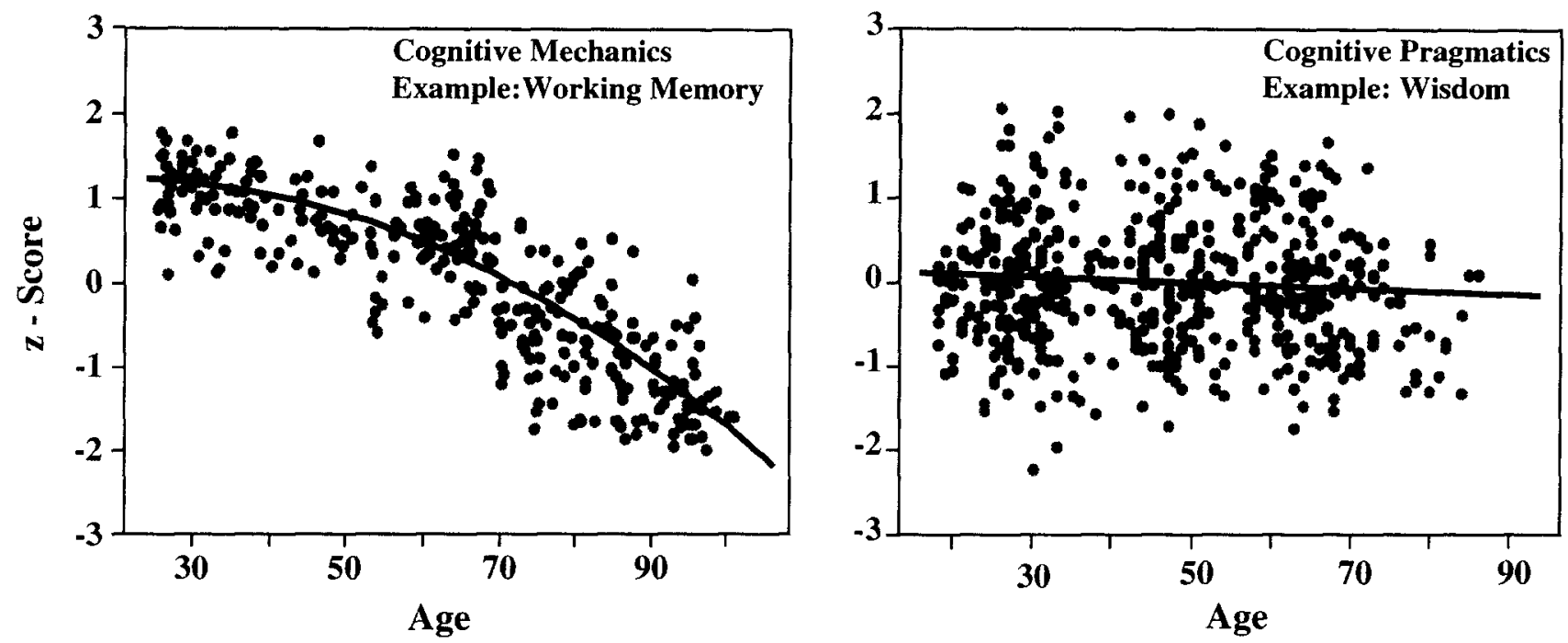

edge that is consistent with this theory of successful development.

\section{Empirical Findings 1: The Role of Chronological Age}

Originally, because of our interest in the search for positive aspects of human aging, our research included a focus on age-comparative analyses of wisdom-related performance (Smith \& Baltes, 1990; Staudinger, 1999a). As for the role of age, Figure 2 presents a summary of four studies with adults. In total, the findings are based on 533 people who ranged in age from 20 to 89 years and represented diverse educational and socioeconomic backgrounds.

Ignoring the possibility of cohort effects, the major finding was that for the age range from about 25 to 75 years of age, the age gradient is zero. The data also tentatively suggested that there may be a limit to the level of wisdomrelated performance in old age, beginning on average around 75 years of age. This finding is understandable in light of studies on the fluid mechanics of cognitive aging. Beyond the age of around 75, one observes a more broadly based decline in cognitive status (Lindenberger \& Baltes, 1997; Schaie, 1996) that is likely to impose increasing "mechanical" limits on level of functioning in response to the kind of wisdom tasks that are used. Recent research with adolescents (Pasupathi, Staudinger, \& Baltes, 1999) has suggested that the major period of acquisition of wisdom-related knowledge and judgment before early adulthood is the age range from about 15 to 25 years.

On the one hand, this finding of no age gradient across most of adulthood may disappoint those who expect, in line with subjective theories of lifespan development, a higher level of functioning in wisdom tasks as people move through midlife into old age. Indeed, if one examines the relative proportion of people in the top $20 \%$ performance category by age across multiple studies, there is some evidence that if age and facilitative experiential contexts collaborate, more older than younger participants are in the top 20\% (P. Baltes, Staudinger, Maercker, \& Smith, 1995; Staudinger, 1999b). This has led us to predict that the "world record" in wisdom may be held by someone in his or her $60 \mathrm{~s}$.

On the other hand, however, the finding of no major age differences during middle adulthood offers support for two of our key assumptions. First, when contrasting findings on the cognitive pragmatics (for which wisdom is a prototype) to research on the fluid mechanics, results indicate that wisdom-related knowledge and judgment are facets of human development that do not show signs of deterioration beginning in earlier stages of adulthood. Second, as we suggested in our developmental causal model of wisdom (Figure 1), having lived longer in itself is not sufficient for acquiring more knowledge and judgment capacity in the wisdom domain. Other factors need to enter into a coalition that, as an ensemble, is generative of wisdom.

\section{Empirical Findings 2: The Role of Professional Experience (Clinical Psychology)}

Another research project involved persons for whom we judged to have experienced a constellation of life processes that were conducive to wisdom. In two studies, clinical psychologists were taken as an example of professional specialization for which both training and practice consist of intensified contact and engagement with questions of 
life planning, life management, and life review (Smith, Staudinger, \& Baltes, 1994; Staudinger, Smith, \& Baltes, 1992). The clinical psychologists were compared with young and old adults of similar educational level and age whose professional training, however, had no particular emphasis in the domain of the fundamental pragmatics of life.

As predicted, clinical psychologists showed higher levels of wisdom-related performance than controls. This was also true for the top range of performances. As we also predicted, however, their performances did not approach expert levels, as judged by our theory-based measurement. On the 7-point scale we developed and applied, the group of clinical psychologists received an average score of 3.8 for the two studies, only slightly above the scale's mean value.

In interpreting this result, one must consider the possibility that it is people with a particular personality constellation and motivational structure who become clinical psychologists. To estimate the contribution of such selection-into-clinical-psychology-profession effects, we examined measures of intelligence and personality. Communality analyses that are based on hierarchical models of regression, which have wisdom-related performance as a dependent variable, allowed us to quantify the joint and separate effects of professional specialization versus intellectual and personality dispositions (Staudinger, Maciel, Smith, \& Baltes, 1998). Professional specialization turned out to be important; in fact, it was the largest unique predictor, accounting for $15 \%$ of the variance in wisdomrelated performance.

\section{Empirical Findings 3: Wisdom-Related Performance Requires the Interplay of Intelligence, Cognitive Style, and Personality}

A major theoretical aspect of our approach (see also Sternberg, 1998) is that wisdom requires and reflects integration and balancing of several spheres of human functioning. On the level of personal characteristics, this includes the orchestration of cognitive and behavioral style and personality attributes. To examine this question, we conducted one study (Staudinger, Lopez, \& Baltes, 1997) in which a large number of relevant measures were considered as predictors of wisdom performance. Specifically, a total of 33 psychometric indicators (that were based on 14 tests) marked the sectors of psychometric intelligence (4 indicators), personality-intelligence interface such as cognitive style and creativity (17 indicators), and personality dispositions (12 indicators). Of these 33 indicators, 10 turned out to be significant predictors of wisdom and were considered in the analyses reported below.

Our general expectation was that none of these measures by themselves would be powerful predictors of wisdom. Moreover, we expected that to achieve a salient prediction, a large number of predictors would be necessary. Finally, we predicted, to test the uniqueness of the wisdom construct, that a significant amount of remaining variance in wisdom-related performance would be predictable only by consideration of parallel tests of wisdom rather than other predictors. The results of hierarchical regression models with follow-up communality analyses supported each of these predictions.

In total, $40 \%$ of the variance in wisdom-related performance could be predicted by considering the 10 significant predictors. First, none of the indicators taken alone, however, accounted for more than $18 \%$ of the variance in wisdom-related ratings. Second, the results showed that even after all (33) of the predictors were brought into the prediction equation, the parallel tests of wisdom-related performance added a relatively large amount of additional variance $(19 \%)$. This finding indicates that even within a differentiated and rather comprehensive psychometric sphere, our wisdom-related measures possessed a high degree of unique variance. Wisdom is more than the ensemble of 33 indicators used to mark the predictor domains of intelligence, personality-intelligence interface, and personality.

What was the specific configuration of predictors of wisdom-related performance? First, there was a significant overlap between all three predictor domains pointing to the coordinative nature of wisdom. Specifically, the predictors from all domains shared $9 \%$ of the predictive variance. Second, the unique prediction of intelligence and personality was relatively small ( $2 \%$ each). Most important, the intelligence-personality interface indicators (e.g., cognitive style, creativity) contributed the largest unique share $(15 \%)$. Within the framework of the interface instruments applied in this study, it was cognitive style (e.g., Sternberg's, 1996, measure of thinking styles) and creativity that particularly showed a positive connection to wisdom-related performance. Among Sternberg's thinking styles, the judicial style (which implies the evaluation and comparison of issues at stake) and the progressive style (which implies moving beyond existing rules and being tolerant of ambiguous situations) were the most salient predictors.

Figure 3 summarizes the main findings from all studies where we examined predictive correlates of wisdom as measured in the Berlin wisdom paradigm. These results indicate that wisdom-related knowledge and judgment are not simply another variant of intelligence or personality. Rather, wisdom implies a coordinating configuration of multiple attributes, including knowledge associated with specific life experiences. The outcome is the orchestration of mind and virtue toward excellence. In this vein, the findings are also in accordance with the results of research on implicit theories of wisdom mentioned above.

\section{Empirical Findings 4: The Study of Persons Nominated as Wise}

It might be supposed that the superior performance of clinical psychologists is less a manifestation of their greater wisdom than of the fact that psychologists fare better than nonpsychologists in a "wisdom theory" developed by members of their own profession. To examine this argument of professional self-enhancing bias, we compared clinical psychologists with other people who were not psychologists but who had been nominated as wise by a 


\section{Figure 3}

The Pattern of Predictive Correlates of Wisdom-Related Performance in Adults

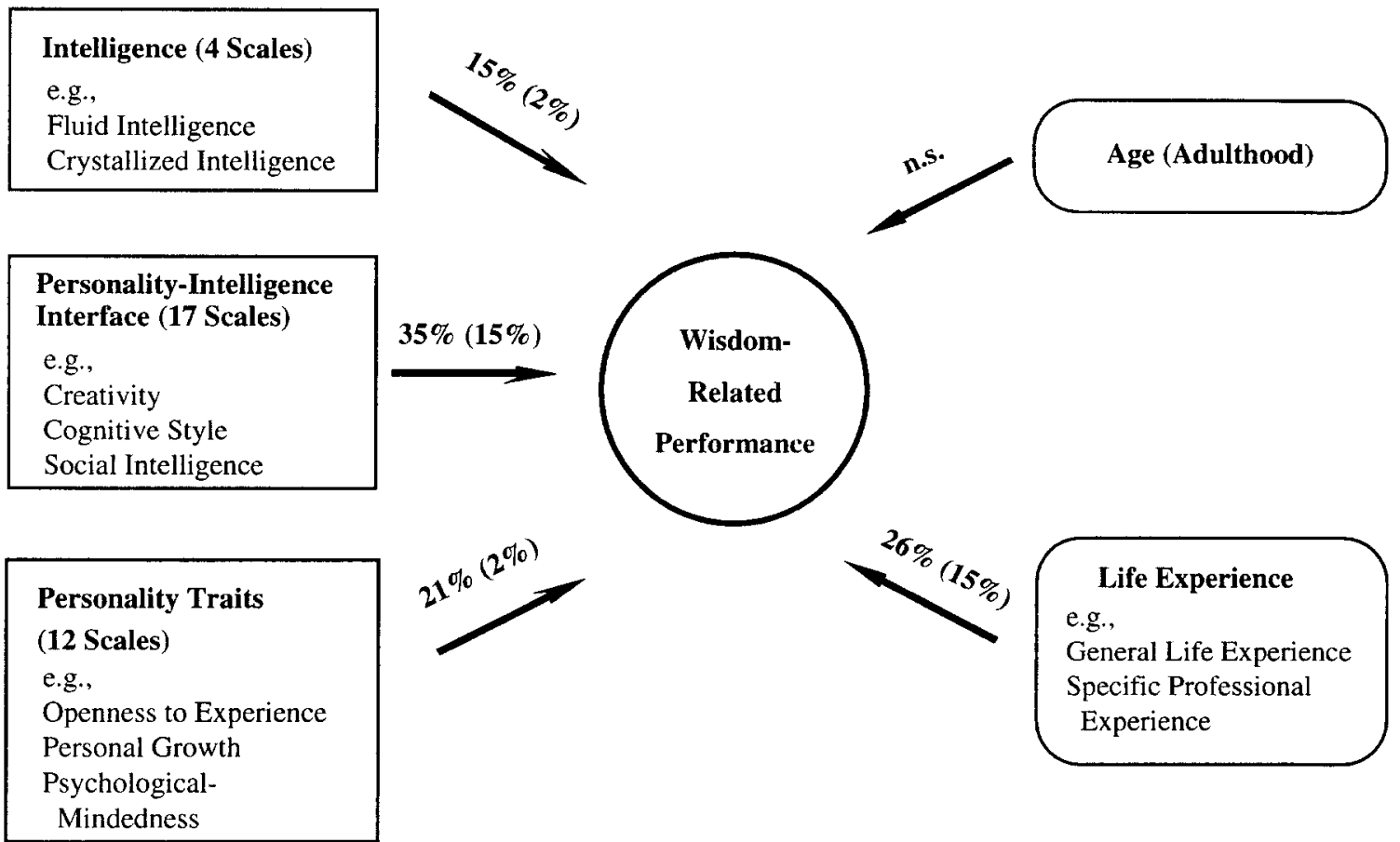

Note. The pattern of predictive correlates of wisdom-related performance in adults illustrates the notion that wisdom is the result of a coalition of multiple sources and attributes orchestrated toward the integration of mind and virtue. Values in parentheses indicate unique predictive contributions that are based on communality analysis (joint representation of data from P. Baltes et al., 1995; Staudinger et al., 1997; Staudinger, Maciel, et al., 1998).

panel of nonpsychologists, independently of our definition of wisdom (P. Baltes et al., 1995).

The wisdom nominees were figures of public distinction who survived an intensive Delphi-like nomination process by a rater panel. Initially, 156 persons were considered. In the end, 21 were chosen as fulfilling the stated criteria. None of the 21 wisdom nominees was a psychologist. Although the range of ages for those nominated as wise was relatively broad (41-79 years), the majority were older adults ( $M=64$ years).

The wisdom-related performance of nominees was compared with clinical psychologists of the same age range and, in addition, with both young (25-35 years) and old control adults $(60-80$ years) who were advanced college graduates but worked outside the field of the professional human services. The participants from all four groups responded to two wisdom-related tasks each. The first task posed a problem of life planning (cf. Smith et al., 1994) and the second was a problem of existential life management involving the potential suicide of a friend (Maercker, Böhmig-Krumhaar, \& Staudinger, 1998).

Overall, wisdom nominees performed at least as well as clinical psychologists and this applied to the top range of performances as well. In fact, for the task most closely tapping into the core of wisdom, that is a task of existential life management, wisdom nominees evinced the highest level of performance, and this included quite a few in the age range from 50 to 70 years. Thus, the conception of wisdom advanced by us is not one where nonpsychologists would not be able to perform well.

\section{Empirical Findings 5: The Inferactive-Minds (Social-Collaborative) Aspect of Wisdom}

One of our central theoretical postures is that wisdom is a collectively anchored product and that individuals by themselves are only "weak" carriers of wisdom (P. Baltes \& Smith, 1990; Staudinger, 1996). To examine the role of collaborative or interactive-minds conditions, we conducted a study in which groups of people performed on wisdom tasks under varying conditions of social support and collaboration. Specifically, we compared people who responded to wisdom tasks alone with (a) those who discussed the problem with a significant other before responding individually, (b) those who engaged in a virtual-internal dialogue about the wisdom problem with a person of their choice, or (c) a group who just had some free time to think about the problem by themselves before responding (Staudinger \& Baltes, 1996a).

The outcome was fully supportive of the view that social collaboration, whether internal or external, facilitates 
wisdom-related performance if, afterwards, persons had the time to reflect about the discourse. This included the condition where the discourse involved an inner voice, that is, private conversations with a person considered by the person to be a model of human functioning. The increase in performance was close to one standard deviation. In line with our interactive-minds and collective approach to wis. dom was the finding that combining individual thinkingtime with an interactive-minds condition was of much importance.

From a lifespan point of view, the finding that, when compared to young adults, older adults benefited more from the actual dialogue condition was especially significant. This is one of the very few findings in research on adult development and aging where older adults profited more from a given intervention than young adults. We interpret this as evidence that with age, when it comes to topics such as wisdom, people acquire a knowledge base that is conducive to input from interpersonal consultation or dialogue.

\section{Empirical Findings 6: Wisdom in Proverbs}

Most recently, we extended our inquiry concerning wisdom to the study of proverbs and their relevance in accessing wisdom-related knowledge (P. Baltes \& Freund, 1999). This research follows the notion of folk psychology (Haselager, 1997; Mieder, 1993) that much of a culture's historically acquired knowledge is stored in proverbs and that accessing this body of knowledge is a major facilitator in achieving and interpreting a good life.

In this instance, we focused on the use of proverbs that reflect the three strategies of life management that Margret Baltes, Paul Baltes, and their colleagues have identified as the foundation to successful life development: selection, optimization, and compensation (M. Baltes \& Carstensen, 1996; P. Baltes, 1997; Freund \& Baltes, in press; Marsiske et al., 1995). Selection involves goals, optimization concerns the means to reach goals, and compensation denotes means that are invoked when established means fail to reach a given goal. Examples of such proverbs are as follows: "Jack of all trades, master of none" (selection), "practice makes perfect" (optimization), and "when there is no wind, grab the oars" (compensation).

To test the availability of such proverb-related knowledge, we presented adults who varied in age from early to late adulthood with life problems that require the use of a particular strategy of problem solving (P. Baltes \& Freund, 1999). We asked adults to choose between two proverbs for each problem situation, one that denoted one of the three key processes (selection, optimization, compensation) and another that described an alternative proverbial strategy (e.g., "everything comes to he who waits").

The outcome was clear and consistent with our expectation that people have access to proverb-based strategies of practical wisdom. Study participants chose the target proverbs of selection, optimization, and compensation more often than the control proverbs. In addition, although older adults typically are much slower in reaction-time tasks, in this instance older adults performed as fast as young adults. We interpret this finding as evidence that with age, people gain wisdom-related knowledge that is captured in proverbs and can be activated when coping with difficult problems of life.

In the future, we intend to extend this line of inquiry in several ways. First, we have plans to examine the degree to which expertise in the use of proverbs correlates with alternative indicators of wisdom such as being nominated as wise, being an effective counselor, or demonstrating high levels of performance in think-aloud wisdom tasks. Furthermore, we plan to conduct experiments in which proverbs are studied that reflect more directly the three metacriteria that we have identified as essential to wisdom: lifespan contextualism, relativism of values and life priorities, and recognition-management of uncertainty. Peng and Nisbett (1999), for example, compared Chinese and American students in relevant work. They studied individual preferences for proverbs that varied in the degree to which they expressed maxim-like prescriptions or reflection-based uncertainty and oppositional information. Chinese students, compared with American students, preferred proverbs that, in line with our conception of wisdom, were more oppositional and contradictory in context.

\section{Wisdom as a Cognitive and Motivational-Emotional Heuristic (Pragmatic) to Orchestrate Mind and Virtue}

In this section, we describe one new line of work that we are initiating to explore another facet of the general conception of wisdom. With this work, we intend to examine more fully to what degree and how wisdom-related knowledge and judgment can serve the function of planning and optimizing human development.

For this purpose, we consider the use of methods associated with the study of cognitive heuristics and pragmatic schemata of reasoning. In general terms, a heuristic can be defined as a highly automatized and organized strategy for directing search processes or for organizing and using information in a certain class of situations (Baron, 1988; Dawes, 1988; Gigerenzer, Todd, \& ABC Research Group, 1999; Haselager, 1997; Kahneman, Slovic, \& Tversky, 1982; Nisbett \& Ross, 1980; Nozick, 1993; Simon, 1983). In the context of the tasks of everyday life, a functionally similar concept is that of a pragmatic in the sense of a pragmatic reasoning schema (Holyoak \& Spellman, 1993; Smith, 1996).

Considering this general approach to the study of behavior, one of us (P. Baltes, 1999) has advanced the idea that one feature of the concept of wisdom is its role as such a heuristic or pragmatic. The direction of the collaborative organization generated by the concept of wisdom would be human excellence in the conduct of life. Specifically, the special focus of this wisdom heuristic would be the activation, organization, and collaborative enlisting of knowledge that directs one's attention to the integration and optimization of mind and virtue. 
What are the definitional elements of this wisdom heuristic? A first set of implications follows from the meaning space of wisdom described above. For example, we suggest that invoking the concept of wisdom coordinates knowledge and judgments about the fundamental pragmatics of life around such properties as (a) strategies and goals involving the conduct and meaning of life; (b) limits of knowledge and uncertainties of the world; (c) excellence of judgment and advice; (d) knowledge with extraordinary scope, depth, and balance; (e) search for a perfect synergy of mind and character; and (f) balancing the good or well-being of oneself and that of others.

There are additional features of the wisdom heuristic (P. Baltes, 1999). One is the role of wisdom in what is called the "binding" problem associated with the issue of integration versus fragmentation of bodies of knowledge. As Stich (1990), for instance, has argued, one of the major deficits of knowledge systems can be their fragmentation or lack of goal- or outcome-oriented binding and collaboration. To counteract such fragmentation of bodies of knowledge, the wisdom heuristic would function as an organizing selector and activator of otherwise more independent bodies of knowledge about the means and ends of a good life.

Other characteristics of the wisdom heuristic are its generality, flexibility, and efficiency in application. Similar to a general purpose heuristic and what Hatano (1988) identified as an adaptive-flexible expertise-related heuristic (compared with routine heuristics), we suggest that the wisdom heuristic has wide applicability. Most issues of the meaning and conduct of life are approachable by this heuristic. Moreover, we submit that the heuristic is highly efficient, considering the complexity of information associated with the domain of the fundamental pragmatics of life. In this vein, and using Gigerenzer's and his colleagues' terminology (Gigerenzer, 1996; Gigerenzer et al., 1999), we would classify the wisdom heuristic as a "fast and frugal" heuristic, as one where within the frame of bounded rationality, highly complex sets of information about the meaning and conduct of life are reduced quickly to their essentials without people being lost in the neverending process of information search that were to occur if wisdom were treated as a case of unbounded rationality.

Finally, we suggest that wisdom is a metaheuristic, that is, a heuristic that organizes, at a high level of aggregation, the pool (ensemble) of bodies of knowledge and commensurate, more specific heuristics that are available to individuals in planning, managing, and evaluating issues surrounding the fundamental pragmatics of life. Such an approach to the psychology of wisdom is consistent with work by philosophers, who, in their search for an interdisciplinarily guided view of the nature of human rationality, have begun to attend to work on heuristics and pragmatic schemata in cognitive psychology; foremost to mention are philosophical pragmatists (Bratman, 1987; Fletcher, 1995; Rorty, 1998; Stich, 1990).

As a cognitive and motivational metaheuristic, then, wisdom is the embodiment of the best "subjective beliefs and laws of life" that a culture and individuals have to offer. Without wisdom as a metaheuristic, individuals' knowledge and judgment about the conduct and meaning of life would be manifested at a lower level of quality, with a greater degree of fragmentation and without the proactive directionality toward optimization that the concept of wisdom prefigures. Moreover, if the wisdom heuristic was acquired systematically and repeatedly over time, the expectation would be that developing individuals would be able to reach more advanced levels of wisdom-related knowledge and judgment than is true to date. It might also be useful to consider the wisdom heuristic in efforts at improving training in clinical, educational, and applied psychology (P. Baltes, 1999; Staudinger \& Baltes, 1996a).

\section{Summary}

In sum, then, we suggest that adding the concept of wisdom to psychological inquiry is a worthwhile challenge. As a concept and as a heuristic, it highlights the jewels and peaks of cultural evolution and human ontogenesis. In its application to human development, wisdom makes explicit the goal of orchestrating mind and virtue toward human excellence and the common good.

There are many open questions, of course. Of much interest is the link of wisdom as a knowledge-based expertise in the fundamental pragmatics of life to actual behavior involving oneself and others. Currently, aside from our work on wisdom nominees and the correlative patterns observed when linking wisdom-related performance to facets of intelligence and personality, there is no relevant empirical evidence to make explicit the link between knowledge and behavior. For example, to what degree do people who excel in our wisdom tasks also demonstrate superior outcomes in their own life management? Is the kind of wisdom-related knowledge and judgment studied by us effective as a life-guiding and life-advancing method? Furthermore, to what degree are people who display wisdom-like knowledge sought out as advisors? What is the behavior they display? These are important questions for future research (e.g., the concept of successful aging in M. Baltes \& Carstensen, 1996, or the concept of art of living in Staudinger, 1999b).

In the ancient history of the concept of wisdom, the sage was often invoked as the only carrier of wisdom, and there were few (Assmann, 1994; P. Baltes, 1999; Hadot, 1995; Oelmüller, 1989). At the same time, it was suggested that sages represent guideposts of excellence for the vast majority of people who themselves would never reach the pinnacle of wisdom. On the one hand, we share in this ancient (e.g., Spinoza) view that wisdom, like "all excellent things, is as difficult as it is rare" (Hadot, 1995, p. 261). On the other hand, when thinking of and about wisdom, individuals are offered a sense of directionality and positive agency. By reference to wisdom, we can participate, for a fleeting moment at least, in the personal utopia of an otherwise unreachable level of excellence.

Elevating the notion of wisdom to an overall life orientation, however, goes beyond the fleeting moment of the present. Making the ensemble of attributes associated with wisdom as explicit as possible, translating it into a more regularly available heuristic (pragmatic), and thereby 
incorporating it into the construction and optimization of human development, individually and collectively, may be a critical step for reaching increasingly higher levels of functioning as the lifespan unfolds. In our view, then, the perennial power of wisdom is its role as a reminder, a source, and a benchmark in the quest for excellence ( $P$. Baltes, 1999).

As a Chinese wisdom proverb says, "Even a very long journey begins with a single step." We add, "And this step is more effective the more it is a step in the right direction." In fact, if the directional movement is correct, such as is true for the direction and destination of wisdom, we can even afford slow progress. To illustrate, we recall a quotation from an ancient Roman (Marc Aurel): "It's better to limp slowly along the right path than walk stridently in the wrong direction." However slow and hard, future work on the psychology of wisdom seems to be a cornerstone of the foundation of what the editors of this special issue claim to be the call for a positive psychology.

\section{REFERENCES}

Agazzi, E. (Ed.). (1991). Science et sagesse: Entretiens de l'Académie Internationale de Philosophie des Sciences, 1990 [Science and wisdom: Proceedings of the International Academy of the Philosophy of Science]. Freiburg, Germany: Universitätsverlag Freiburg.

Alexander, C. N., \& Langer, E. J. (Eds.). (1990). Higher stages of human development. New York; Oxford University Press.

Anderson, J. R. (1987). Skill acquisition: Compilation of weak-method problem solutions. Psychological Review, 94, 192-210.

Arlin, P. K. (1993). Wisdom and expertise in teaching: An integration of perspectives. Leaming and Individual Differences, 5, 341-349.

Assmann, A. (1994). Wholesome knowledge: Concepts of wisdom in a historical and cross-cultural perspective. In D. L. Featherman, R. M. Lerner, \& M. Perlmutter (Eds.), Life-span development and behavior (Vol. 12, pp. 187-224). Hillsdale, NJ: Erlbaum.

Baltes, M. M., \& Carstensen, L. L. (1996). The process of successful ageing. Ageing and Society, 16, 397-422.

Baltes, P. B. (1987). Theoretical propositions of life-span developmental psychology: On the dynamics between growth and decline. Developmental Psychology, 23, 611-626.

Baltes, P. B. (1993). The aging mind: Potential and limits. Gerontologist, 33, 580-594.

Baltes, P. B. (1997). On the incomplete architecture of human ontogeny: Selection, optimization, and compensation as foundation of developmental theory. American Psychologist, 52, 366-380.

Baltes, P. B. (1999). Wisdom: The orchestration of mind and virtue. Manuscript in preparation, Max Planck Institute for Human Development, Berlin.

Baltes, P. B., Dittmann-Kohli, F., \& Dixon, R. A. (1984). New perspectives on the development of intelligence in adulthood: Toward a dual-process conception and a model of selective optimization with compensation. In P. B. Baltes \& J. O. G. Brim (Eds.), Life-span development and behavior (Vol, 6, pp. 33-76). New York: Academic Press.

Baltes, P. B., \& Freund, A. M. (1999). SOC-related knowledge about iffespan development: It's in the proverbs. Unpublished manuscript, Max Planck Institute for Human Development, Berlin.

Baltes, P. B., \& Smith, J. (1990). The psychology of wisdom and its ontogenesis. In R. J. Sternberg (Ed.), Wisdom: Its nature, origins, and development (pp. 87-120). New York: Cambridge University Press.

Baltes, P. B., Smith, J., \& Staudinger, U. M. (1992). Wisdom and successful aging. In T. Sonderegger (Ed.), Nebraska Symposium on Motivation (Vol. 39, pp. 123-167). Lincoln: University of Nebraska Press.
Baltes, P. B., \& Staudinger, U. M. (1993). The search for a psychology of wisdom. Current Directions in Psvchological Science, 2, 75-80.

Baltes, P. B., \& Staudinger, U. M. (1998). Wisdom. In H. Friedman (Ed.), Encyclopedia of mental health (Vol. 3, pp. 699-706). San Diego, CA: Academic Press.

Baltes, P. B., Staudinger, U. M., \& Lindenberger, U. (1999). Lifespan psychology: Theory and application to intellectual functioning. Annual Review of Psychology, 50, 471-507.

Baltes, P. B., Staudinger, U. M., Maercker, A., \& Smith, J. (1995). People nominated as wise: A comparative study of wisdom-telated knowledge. Psychology and Aging, 10, 155-166.

Bandura, A. (1982). The psychology of chance encounters and life paths. American Psychologist, 37, 747--755

Baron, J. (1988). Thinking and deciding. Cambridge, MA: Cambridge University Press.

Basseches, M. (1984). Dialectical thinking and adult development. Norwood, NJ: Ablex.

Birren, J. E. \& Fisher, L. M. (1990). The elements of wisdom: Overview and integration. In R. J. Sternberg (Ed.), Wisdom: Its nature, origins, and development (pp. 317-332). New York: Cambridge University Press.

Blanchard-Fields, F., \& Hess, T. M. (Eds.). (1996). Perspectives on cognitive change in adulthood and aging. New York: McGraw-Hill.

Bloom, B. 1 1985). Developing talent in voung people. New York: Ballantine.

Bratman, M. E. (1987). Intention, plats and practical reason. Cambridge, MA: Harvard University Press.

Carstensen, L. L. (1995). Evidence for a life-span theory of socioemotional selectivity. Current Directions in Psychological Science, 4, 151156.

Clayton, V. P., \& Birren. J. E. (1980). The development of wisdom across the life span: A reexamination of an ancient topic. In P. B. Baltes \& J. O. G. Brim (Eds.), Life-span development and behavior (Vol. 3, pp. 103-135). New York: Academic Press

Cole, M. (1996). Interacting minds in a life-span perspective: A cultural/ historical approach to culture and cognitive development. In P. B. Baltes \& U. M. Staudinger (Eds.), Interactive minds: Life-span perspectives on the social foundation of cognition (pp. 59-87). New York: Cambridg̣e University Press.

Cook, A. (1993). Canons and wisdom. Philadelphia: University of Pennsylvania Press.

Csikszentmihalyi, M., \& Rathunde, K. (1990). The psychology of wisdom: An evolutionary interpretation. In R. J. Sternberg (Ed.), Wisdom: Its nature, origins, and development (pp. 25-51). New York: Cambridge University Press.

Dawes, R. M. (1988). Rational choice in an uncertain world. Orlando, FL: Harcourt Brace Jovanovich.

Dittmann-Kohli, F., \& Baltes. P. B. (1990). Toward a neofunctionalist conception of adult intellectual development: Wisdon as a prototypical case of intellectual growth. In C. Alexander \& E. Langer (Eds.), Higher stages of human development: Perspectives on adult growth (pp. 5478). New York: Oxford University Press.

Dixon, R. A., \& Baltes, P. B. (1986). Toward life-span research on the functions and pragmatics of intelligence. In R. J. Sternberg \& R. K. Wagner (Eds.), Practical intelligence: Nature and origins of competence in the everyday world (pp. 203-234). New York: Cambridge University Press.

Dörner, D. (1983). Heuristics and cognition in complex systems. In R. Groner, M. Groner, \& W. F. Bischof (Eds.), Methods of heuristics (pp. 89-107). Hillsdale. NJ: Erlbaum.

Ericsson, K. A., \& Lehmann, A. C. (1996). Expert and exceptional performance: Evidence of maximal adaptation to task constraints. Annual Retien of Psychology, 47. 273-305.

Ericsson, K. A., \& Simon, H. A. (1984). Protocol analysis: Verbal reports as data. Cambridge, MA: MIT Press.

Erikson, E. H. (1959). Identity and the life cycle. New York: International University Press.

Fletcher, G. J. O. (1995). The scientific credibility of folk psychology. Mahwah. NJ: Erlbaum

Freund, A. M., \& Baltes, P. B. (in press). The orchestration of selection, optimization, and compensation: An action-theoretical conceptualization of a theory of developmental regulation. In W. J. Perrig \& A. Grob 
(Eds.), Control of human behavior, mental processes and consciousness. Hillsdale, NJ: Erlbaum.

Gigerenzer, G. (1996). Rationality: Why social context matters. In P. B Baltes \& U. M. Staudinger (Eds.), Interactive minds: Life-span perspectives on the social foundation of cognition (pp. 317-346). New York: Cambridge University Press.

Gigerenzer, G., Todd, P., \& ABC Research Group. (1999). Simple heu ristics that make us smart. New York: Oxford University Press.

Hadot, P. (1995). Philosophy as a way of life. Boston: Blackweil.

Hall, G. S. (1922). Senescence: The last half of life. New York: Appleton.

Haselager, W. F. G. (1997). Cognitive science and folk psychology: The right frame of mind. London, UK: Sage.

Hatano, G. (1988). Social and motivational bases for mathematical understanding. In G. B. Saxe \& M. Gearhart (Eds.), Children's mathematics (pp. 55-70). San Francisco: Jossey-Bass.

Holliday, S. G., \& Chandler, M. J. (1986). Wisdom: Explorations in adult competence. In J. A. Meacham (Ed.), Contributions to human development (Vol. 17, pp. 1-96). Basel, Switzerland: Karger.

Holyoak, K. J., \& Spellman, B. A. (1993). Thinking. Annual Review of Psychology, 44, 265-315.

Kahneman, D., Slovic, P., \& Tversky, A. (Eds.). (1982). Judgment under uncertainty: Heuristics and biases. Cambridge, MA: Cambridge University Press.

Kekes, J. (1995). Moral wisdom and good lives. Ithaca, NY: Cornell University Press.

Kitchener, K. S., \& Brenner, H. G. (1990). Wisdom and reflective judgement: Knowing in the face of uncertainty. In R. J. Sternberg (Ed.) Wisdom: Its nature, origins, and development (pp. 212-229). New York: Cambridge University Press.

Labouvie-Vief, G. (1990). Wisdom as integrated thought: Historical and developmental perspectives. In R. J. Sternberg (Ed.), Wisdom: Its nature, origins, and development (pp. 52-83). New York: Cambridge University Press.

Labouvie-Vief, G. (1995). Psyche and eros: Mind and gender in the life course. New York: Cambridge University Press.

Lehrer, K., Lum, B. J., Slichta, B. A., \& Smith, N. D. (Eds.). (1996) Knowledge, teaching and wisdom. Dordrecht, Netherlands: Kluwer.

Lindenberger, U., \& Baltes, P. B. (1997). Intellectual functioning in old and very old age: Cross-sectional results from the Berlin Aging Study. Psychology and Aging, 12, 410-432.

Maercker, A., Böhmig-Krumhaar, S. A., \& Staudinger, U. M. (1998) Existentielle Konfrontation als Zugang zu weisheitsbezogenem Wissen und Urteilen: Eine Untersuchung von Weisheitsnominierten [Existential confrontation as access to wisdom-related knowledge and judgment: A study of wisdom nominees]. Zeitschrift für Entwicklungspsychologie und Pädagogische Psychologie, 30, 2-12.

Marsiske, M., Lang, F. R., Baltes, M. M., \& Baltes, P. B. (1995). Selective optimization with compensation: Life-span perspectives on successful human development. In R. A. Dixon \& L. Bäckman (Eds.), Compensation for psychological deficits and declines: Managing losses and promoting gains (pp. 35-79). Hillsdale, NJ: Erlbaum.

Maxwell, N. (1984). From knowledge to wisdom. New York: Basil Blackwell.

McAdams, D. P., \& de St. Aubin, E. (Eds.). (1998). Generativity and adult development: How and why we care for the next generation. Washington, DC: American Psychological Association.

Mieder, W. (1993). Proverbs are never out of season: Popular wisdom in the modern age. New York: Oxford University Press.

Nichols, R. (1996). Maxims, "practical wisdom," and the language of action. Political Theory, 24, 687-705

Nisbett, R. E., \& Ross, L. (1980). Human inference: Strategies and shortcomings of social judgment. Englewood Cliffs, NJ: Prentice Hall.

Nozick, R. (1993). The nature of rationality. Princeton, NJ: Princeton University Press.

Oelmüller, W. (1989). Philosophie und Weisheit [Philosophy and wisdom]. Paderborn, Germany: Schöningh

Orwoll, L., \& Perlmutter, M. (1990). The study of wise persons: Integrating a personality perspective. In R. J. Sternberg (Ed.), Wisdom: Its nature, origins, and development (pp. 160-177). New York: Cambridge University Press.

Pasupathi, M., \& Baltes, P. B. (in press). Wisdom. In A. E. Kazdin (Ed.), Encyclopedia of psychology. New York: Oxford University Press.
Pasupathi, M., Staudinger, U. M., \& Baltes, P. B. (1999). The emergence of wisdom-related knowledge and judgment during adolescence. Berlin: Max Planck Institute for Human Development.

Peng, K., \& Nisbett, R. E. (1999). Culture, dialectics, and reasoning about contradiction. American Psychologist, 54, 741-754.

Rice, E. F., Jr. (1958). The renaissance idea of wisdom. Cambridge, MA: Harvard University Press.

Riegel. K. F. (1973). Dialectical operations: The final period of cognitive development. Human Development, 16, 346-370.

Robinson, D. N. (1990). Wisdom through the ages. In R. J. Sternberg (Ed.), Wisdom: Its nature, origins, and development (pp. 13-24). New York: Cambridge University Press.

Rorty, R. (1998). Truth and progress: Philosophical papers. New York: Cambridge University Press.

Rudolph, K. (1987). Wisdom. In M. Eliade (Ed.), Encyclopedia of religion: Wisdom (Vol. 15, pp. 393-401). New York: Macmillan.

Schaie. K. W. (1996). Adult intellectual development: The Seattle longitudinal study. New York: Cambridge University Press.

Shweder, R. A. (1991). Thinking through cultures. Cambridge, MA: Harvard University Press.

Simon, H. A. (1983). Reasoning in human affairs. Stanford, CA: Stanford University Press.

Smith, J. (1996). Planning about life: A social-interactive and life-span perspective. In P. B. Baltes \& U. M. Staudinger (Eds.), Interactive minds: Life-span perspectives on the social foundation of cognition (pp. 242-275). New York: Cambridge University Press.

Smith, J., \& Baltes, P. B. (1990). A study of wisdom-related knowledge: Age/cohort differences in responses to life planning problems. Developmental Psychology, 26, 494-505.

Smith, J., Staudinger, U. M., \& Baltes, P. B. (1994). Occupational settings facilitating wisdom-related knowledge: The sample case of clinical psychologists. Journal of Consulting and Clinical Psychology, 62, 989-999.

Sowarka, D. (1989). Weisheit und weise Personen: Common-SenseKonzepte älterer Menschen [Wisdom and wise persons: Common-sense views by elderly people]. Zeitschrift für Entwicklungspsychologie und Pädagogische Psychologie, 21, 87-109.

Staudinger, U. M. (1996). Wisdom and the social-interactive foundation of the mind. In P. B. Baltes \& U. M. Staudinger (Eds.), Interactive minds: Life-span perspectives on the social foundation of cognition ( $\mathrm{pp}$. $276-315)$. New York: Cambridge University Press.

Staudinger, U. M. (1999a). Older and wiser? Integrating results on the relationship between age and wisdom-related performance. International Journal of Behavioral Development, 23, 641-664.

Staudinger, U. M. (1999b). Social cognition and a psychological approach to an art of life. In F. Blanchard-Fields \& B. T. Hess (Eds.), Social cognition, adult development, and aging (pp. 343-375). New York: Academic Press.

Staudinger, U. M., \& Baltes, P. B. (1994). Psychology of wisdom. In R. J. Stermberg (Ed.), Encyclopedia of intelligence (Vol. 1, pp. 143-152). New York: Macmillan.

Staudinger, U. M., \& Baltes, P. B. (1996a). Interactive minds: A facilitative setting for wisdom-related performance? Journal of Personality and Social Psychology, 71, 746-762.

Staudinger, U. M., \& Baltes, P. B. (1996b). Weisheit als Gegenstand psychologischer Forschung [Wisdom as a topic of psychological research]. Psychologische Rundschau, 47, 57-77.

Staudinger, U. M., Lopez, D., \& Baltes, P. B. (1997). The psychometric location of wisdom-related performance: Intelligence, personality, and more? Personality and Social Psychology Bulletin, 23, 1200-1214

Staudinger, U. M., Maciel, A. G., Smith, J., \& Baltes, P. B. (1998). What predicts wisdom-related performance? A first look at personality, intelligence, and facilitative experiential contexts. European Journal of Personality, 12, 1-17.

Staudinger, U. M., Raykov, T., Böhmig-Krumhaar, S., \& Baltes, P. B. (1999). How general are domain-specific judgmental strategies? The sample case of wisdom-related performance. Berlin: Max Planck Institute for Human Development.

Staudinger, U. M., Smith, J., \& Baltes, P. B. (1992). Wisdom-related knowledge in a life review task: Age differences and the role of professional specialization. Psychology and Aging, 7, 271-281. 
Sternberg, R. J. (1985). Implicit theories of intelligence, creativity, and wisdom. Journal of Personality and Social Psychology, 49, 607-627.

Sternberg, R. J. (1986). Intelligence, wisdom, and creativity: Three is better than one. Educational Psychologist, 21, 175-190.

Sternberg, R. J. (Ed.). (1990). Wisdom: Its nature, origins, and development. New York: Cambridge University Press.

Sternberg, R. J. (1996). Styles of thinking. In P. B. Baltes \& U. M. Staudinger (Eds.), Interactive minds: Life-span perspectives on the social foundation of cognition (pp. 347-365). New York: Cambridge University Press.
Stemberg, R. J. (1998). A balance theory of wisdom. Review of General Psychology, 2, 347-365.

Stich, S. P. (Ed.). (1990). The fragmentation of reason: Preface to a pragmatic theory of cognitive evaluation. Cambridge, MA: MIT Press.

Taranto, M. A. (1989). Facets of wisdom: A theoretical synthesis. International Journal of Aging and Human Development, 29, 1-21.

Welsch, W. (1995). Vermunft: Die zeitgenössische Vernunftkritik und das Konzept der transversalen Vernunft [Practical reason: A modern critique of reason and the concept of transversal reasoning]. Frankfurt am Main, Germany: Suhrkamp.

\section{Appendix A \\ General Criteria Derived From Cultural-Historical Analysis and Specific Criteria (Berlin Wisdom Paradigm) Used to Analyze Wisdom-Related Products}

\section{General Criteria Outlining the Nature of Wisdom}

Wisdom addresses important and difficult questions and strategies about the conduct and meaning of life.

Wisdom includes knowledge about the limits of knowledge and the uncertainties of the world.

Wisdom represents a truly superior level of knowledge, judgment, and advice.

Wisdom constitutes knowledge with extraordinary scope, depth, measure, and balance.

Wisdom involves a perfect synergy of mind and character, that is, an orchestration of knowledge and virtues.

Wisdom represents knowledge used for the good or well-being of oneself and that of others.

Wisdom is easily recognized when manifested, although difficult to achieve and to specify.

\section{Criteria Used in Berlin Wisdom Paradigm to Operationalize Wisdom as Expertise in the Fundamental Pragmatics of Life}

Rich factual knowledge about life

Rich procedural knowledge about life

Lifespan contextualism

Relativism of values and life priorities

Recognition and management of uncertainty

(Appendixes continue) 


\section{Appendix B}

\section{Berlin Wisdom Paradigm: Illustration of a Wisdom-Related Task With Examples of Extreme Responses (Abbreviated)}

A 15-year-old girl wants to get married right away. What should one/she consider and do?

\section{Low Wisdom-Related Score}

A 15-year-old girl wants to get married? No, no way, marrying at age 15 would be utterly wrong. One has to tell the girl that marriage is not possible. (After further probing) It would be irresponsible to support such an idea. No, this is just a crazy idea.

\section{High Wisdom-Related Score}

Well, on the surface, this seems like an easy problem. On average, marriage for 15-yearold girls is not a good thing. But there are situations where the average case does not fit. Perhaps in this instance, special life circumstances are involved, such that the girl has a terminal illness. Or the girl has just lost her parents. And also, this girl may live in another culture or historical period. Perhaps she was raised with a value system different from ours. In addition, one has to think about adequate ways of talking with the girl and to consider her emotional state. 